\title{
IMPLEMENTING BINARY SEARCH TREE CONCEPT FOR IMAGE CRYPTOGRAPHY
}

\author{
Mohammed A. F. Al-Husainy ${ }^{1}$ and Hamza A. A. Al-Sewadi ${ }^{2}$ \\ ${ }^{1}$ Department of Computer Science, Middle East University, Amman, Jordan \\ ${ }^{2}$ Department of Communication Engineering, Iraq University College, Iraq \\ ${ }^{1}$ mal-husainy@meu.edu.jo, ${ }^{2}$ alsewadi46@gmail.com
}

\begin{abstract}
So many cryptographic systems are secure and in use today, such as AES, 3DES, Blowfish, RC4, etc., however, new ideas and innovations are still highly required due to the ever-growing threats to data security. This paper presents a novel idea for symmetric image encryption utilizing binary search tree mechanism for generating the encryption/decryption key. This key consists of local and global parts with free user controlled key length, hence, it provides good flexibility for high security, which is manifested in the encrypted images. For every single byte of the encrypted image, the substitution and transposition operations involve all the image contents, fulfilling Shannon's diffusion and confusion concept. Experimental computations of the proposed encryption method have shown satisfactory visual and image energy distribution histogram. Besides, the Peak Signal to Noise Ratio (PSNR) and Normalized Mean Absolute Error (NMAE) measurement results were comparable with the widely used cryptosystems such as DES and AES. On the other hand, the proposed method has flexible key length and shorter execution time by $10 \%$ or more depending on the image contents.
\end{abstract}

Keywords - Avalanche effect; security; image encryption

\section{INTRODUCTION}

Growing cyber security attacks are the main worry of all people around the world. Efforts for enhancing data security measures are continuing by academics and industrial firms paralleled with continuous digging for security breaches by hacking and criminal actions, individuals, and groups. So many cryptography systems are in use today that are secure enough, such as AES, 3DES, Blowfish, RC4, etc., [1, 2]. However, academic and industrial research teams are continuously looking for new innovative techniques or enhancing previously developed ones. These efforts are spent in order to protect the everincreasing torrent of sensitive personal computers, governmental, and commercial data that either being stored in various integrated computers or being in transit over various digital communication means.

Although data encryption/decryption techniques are the ancient solutions designed to protect information from intruders since thousands of years, they are still the most widely used techniques. Historically, only symmetric systems were available, where the same secret key is used for encryption and decryption processes. However, recently since 1976, at the age of digital computers, asymmetric systems were developed, where two or more different but related keys are used; one for encryption and another for decryption processes. These techniques are referred to as public-key systems [3]. Symmetric systems are comparatively fast and more difficult to break, but they have the serious problem of key

Received: May 23, 2019

Reviewed: August 21, 2019

Accepted: September 9, 2019 
distribution, while the asymmetric systems are slower and less secure, but proved convenient for authentication and key distribution [4].

In this paper, a novel technique is suggested for symmetric image encryption using a key generated by a binary search tree method. After this brief definition, literature review presented in Sections 2, then, the methodology of the proposed image cryptography scheme is described in Section 3. Section 4 lists out the experimentation, results, and discussion. Then Section 5 concludes the work.

\section{RELATED WORK}

So many techniques for image cryptography algorithms have been reported and implemented, however, only some examples will be listed here as representative of binary tree cryptographic algorithms, that triggered the author's current research. In 2007, Lim and Mun [5] proposed a packet classification algorithm applying binary search on prefix length to the area-based quad-tree. Their algorithm relies on constructing multiple disjoint trees based on the relative hierarchical level rule, hence avoiding the pre-computation required in the binary search on length. They also suggested two new optimization techniques based on rule priorities. In their test, they implemented 5000 different rules and tested the memory consumption and number of bytes per rule. They showed that the performance of the algorithm was steady and does not depend on the table characteristics.

In 2010, Wie and Zang [6] proposed a Key Insertion and Splay Tree encryption algorithm (referred to as KIST), that uses asynchronous key sequence to change the tree dynamically and secretly. The encryption was achieved by byte XORing and swapping. The proposed algorithm suffers several disadvantages and limitations, such as bad error propagation, which is good for message integrity, but not useful in a noisy channel. Besides, no resynchronizing method in the key sequence was implemented. Then, in 2012, Saraswathi and Venkatesulu [7] proposed a naive algorithm for encrypting any multimedia content using blocks of bits rather than bytes or pixels. It can encrypt any type of compressed multimedia content by random substitution using Binary Tree Traversal (BTT), row shifting and column shifting. They claimed to obtain superior performance compared with DES algorithm, and its suitability for encrypting audio, images, video, and text data.

For the protection of data stored in the cloud, an encryption algorithm was proposed by Vanaga in 2014 [8], aiming to meet the security and privacy issues in cloud warehouses. In this algorithm. message contents are converted to ASCII codes, building a binary tree from these values and rearrange pre-order and in-order. Although it was convenient for cloud computing application, the time complexity was a problem. Then in 2015, Sharma and Bhatt [9] reported a binary tree concept for block cipher encryption with considerably increased computation complexity and security. In this algorithm, any input message is first broken into blocks of the 8 characters each, which are assigned to the leaf nodes of the binary tree of level 3, and other nodes of the binary tree are then filled using functions that generate some characters corresponding to the internal nodes of the binary tree. Then another function is applied to transpose the positions of the characters in binary tree. They claim cipher that provides high security in various applications; however, the ciphertext size is double the plaintext size in their algorithm. Also, in 2015, Sagar [10] proposed an encryption algorithm for text message implementing tree traversal processes. He relied on the pre-order, in-order and post-order processes for encryption and decryption. Although, this algorithm reflects the person's interest in the use of binary tree in cryptography, it was too simple and trivial.

In 2017, Priya et al. [11] suggested and applied a novel encryption algorithm that focuses on four block ciphers based complete encryption technique. It implements a binary tree traversal for multi-bit word parallelism using substitution and a two-dimensional array to perform a nonlinear diffusion process. Improvements were observed in the required 
memory size, encryption time, and CPU utilization, besides obtaining high security as compared with existing algorithms such as AES, RC5, and RC6. Also, in the same year Sivakumar et al. [12] included Binary Tree Traversal (BTT) process as one level in a data encryption and decryption algorithm that also comprises of ASCII code conversion. BTT is used to achieve permutation and ASCII codes for substitution. However, the algorithm was simple and would be useful for limited applications only. Moreover, Amounas [13] presented a new approach to enhance the security of Amazigh text using elliptic curve cryptography and tree traversal technique. The Unicode representation of the Amazigh text is scrambled first using the tree traversal method and then implemented in the elliptic curve cryptosystem. Experimental results have shown that involving the tree traversal process has improved the efficiency of the ECC algorithm.

This paper reports a novel idea for symmetric image encryption utilizing binary search tree mechanism to create a strong key to be used for image encryption/decryption processes. This key consists of local and global parts with free user controlled key length seeking good flexibility for high security. In the following, the idea of binary search tree is briefly outlined and how it can be implemented to be used in the rest of the paper.

\section{BINARY SEARCH TREE}

The shape of any generated Binary search tree depends mainly on the sequence of the values that are inserted in the nodes in that tree. To clarify this property of the Binary search tree, Figure 1 depicted two examples for two different binary trees with ten nodes having the values $0 \ldots 9$. The node labels are arranged randomly as the sequence is of no significance here.

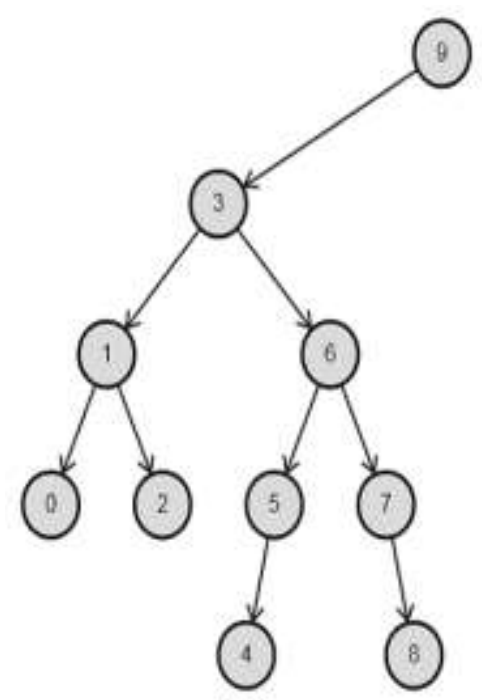

(a)

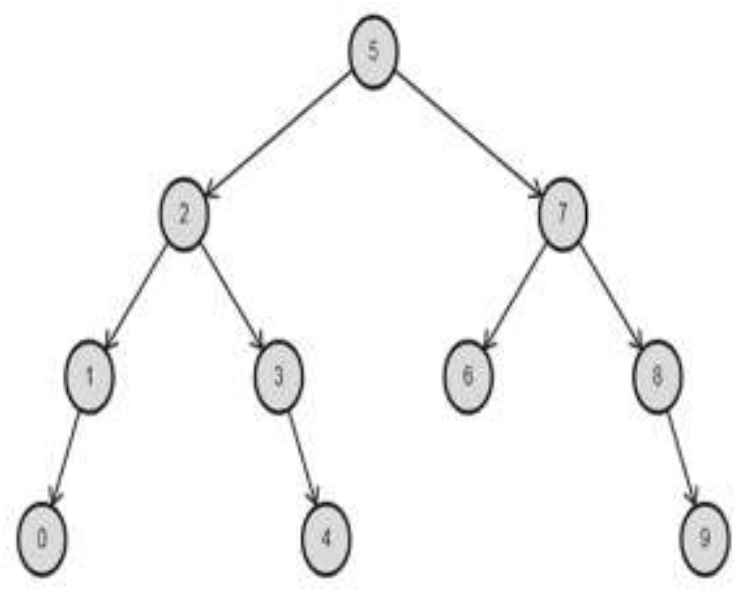

(b)

Fig. 1 Two different Binary trees generated from two different sequences of values (a) 9 ,

$$
3,6,1,5,7,0,4,2,8 \text { and (b) 5, 2, 3, 7, 1, 6, 8, 0, 9, } 4 \text {. }
$$

In Figure 1, the path from each node in the tree to the root contains different number of nodes that contained different values. Table I contains the list of the node values in the path from each node to the root (exclude the node itself). 
Table I. List of Values in the Path from each Node to the Root (Exclude the Node Itself)

\begin{tabular}{|l|l|l|}
\hline \multirow{2}{*}{ Node } & \multicolumn{2}{|l|}{ List of values in the path of the node } \\
\cline { 2 - 3 } & \multicolumn{1}{|c|}{ in tree (a) } & \multicolumn{1}{c|}{ in tree (b) } \\
\hline$N_{0}$ & $1,3,9$ & $1,2,5$ \\
\hline$N_{1}$ & 3,9 & 2,5 \\
\hline$N_{2}$ & $1,3,9$ & 5 \\
\hline$N_{3}$ & 9 & 2,5 \\
\hline$N_{4}$ & $5,6,3,9$ & $3,2,5$ \\
\hline$N_{5}$ & $6,3,9$ & - \\
\hline$N_{6}$ & 3,9 & 7,5 \\
\hline$N_{7}$ & $6,3,9$ & 5 \\
\hline$N_{8}$ & $7,6,3,9$ & 7,5 \\
\hline$N_{9}$ & - & $8,7,5$ \\
\hline
\end{tabular}

\section{THE PROPOSED IMAGE ENCRYPTION METHOD}

The main idea of the proposed encryption method is to focus on utilizing the structure of the Binary search tree as sub keys that are constructed from the input key for image encryption and decryption processes.

In the encryption phase, the inputs are the source image $\boldsymbol{S}$ and the secret key $\boldsymbol{K}$, which might be any type of digital files, and produces the encrypted image $\boldsymbol{E}$ as output. Both $\boldsymbol{S}$ and $\boldsymbol{K}$ are treated as files of bytes. Where $\boldsymbol{S}_{\text {Length }}$ and $\boldsymbol{K}_{\text {Length }}$ represent the length in bytes of $\boldsymbol{S}$ and $\boldsymbol{K}$ respectively. Four main processes are conducted through the encryption phase: 1) Initial Key Generation, 2) Global Image Processing, 3) Local Image Processing, and 4) Construct an Encrypted Image $\boldsymbol{E}$. Each process consists of a set of operations as detailed in the following

\subsection{INITIAL KEY GENERATION}

Read the contents (bytes) of the secret key $\boldsymbol{K}$ and implement equation 1 to generate the specific key used by the proposed encryption method.

$$
\boldsymbol{K}_{\boldsymbol{i}}=\boldsymbol{K}_{\boldsymbol{i}} \oplus \boldsymbol{K}_{\boldsymbol{j}} \quad \text { for } i, j: 0 \text { to } K_{\text {Length }} \text { and } i \neq j
$$

Where $\oplus$ represents the XOR operation and $i$ and $\boldsymbol{j}$ represent the $i^{t h}$ and $j^{\text {th }}$ bytes in the secret key $\boldsymbol{K}$ respectively.

\subsection{GLOBAL IMAGE PROCESSING}

In this step, a set of operations are implemented on the image and key, each of the source image $\boldsymbol{S}$ and the key $\boldsymbol{K}$ are treated as one block (without splitting $\boldsymbol{S}$ or $\boldsymbol{K}$ into blocks).

\subsubsection{PREPARATION OPERATION}

The following steps are followed in order to re-represent the secret key $\boldsymbol{K}$ to construct a new key $\boldsymbol{K}^{\prime}$.

1. Equation 2 is used to calculate the minimum number of bits $N$ that are needed to represent the index of each byte in the source image $\boldsymbol{S}$. The indices of the byte in the source image $\boldsymbol{S}$ are from $\mathbf{0}$ to $\boldsymbol{S}_{\text {Length }} \mathbf{- 1}$.

$$
2^{N} \geq S_{\text {Length }}
$$

2. Represent the secret key $\boldsymbol{K}$ as a list of bits $\boldsymbol{K} \boldsymbol{B}$ by converting each byte of $\boldsymbol{K}$ into its equivalent bits. The length of $\boldsymbol{K B}$ is calculated using equation 3.

$$
\boldsymbol{K B}_{\text {Length }}=\boldsymbol{K}_{\text {Length }} \times 8
$$


3. Construct a new key $\boldsymbol{K}^{\prime}$ by sequentially reading $\boldsymbol{N}$ bits from $\boldsymbol{K B}$, converting them to their equivalent decimal values, then store these values in $\boldsymbol{K}^{\prime}$. Ignore any remaining number of bits less than $\boldsymbol{N}$ at the end of $\boldsymbol{K B}$.

4. Implement equation 4 on each value in $\boldsymbol{K}^{\prime}$ to create new values that are limited in the range $\mathbf{0}$ to $\boldsymbol{S}_{\text {Length }} \mathbf{- 1}$.

$$
\boldsymbol{K}_{j}^{\prime}=\boldsymbol{K}_{j}^{\prime} \bmod \left(S_{\text {Length }}-1\right)
$$

Where $j$ represents the index of $\boldsymbol{K}^{\prime}$ and $j: 0 \ldots \boldsymbol{K}_{\text {Length }}^{\prime}-1$.

4.2.2 GLOBAL KEY GENERATION (BINARY SEARCH TREE): After completing the previous preparation operation, the following steps are implemented to generate the Binary search tree (in (1)) that will be used (in (2)) to extract the necessary lists to be used later in the next operations.

1. Read sequentially the distinct values of $\boldsymbol{K}^{\prime}$, without repetition, then generate the corresponding Binary search tree from them. The generated Binary search tree must contain $\boldsymbol{S}_{\text {Length }}$ nodes; each node contains one value in the range $\mathbf{0}$ to $\boldsymbol{S}_{\text {Length }}$. If some values haven't been found in $\boldsymbol{K}^{\prime}$, then these missed values must be inserted to the Binary search tree after finishing the read operation of the $\boldsymbol{K}^{\prime}$ values.

2. Extract the lists $\boldsymbol{L}_{\mathbf{0}}$ to $\boldsymbol{L}_{S L e n g t h}$ of values in the path of each node from $\boldsymbol{N}_{\mathbf{0}}$ to $\boldsymbol{N}_{\text {SLength }}$, respectively in the generated Binary search tree in (1) [where $N_{i}$ is the number of bits in $\boldsymbol{K}^{\prime}$ and $\boldsymbol{L}_{\boldsymbol{i}}$ are the list of values in $\boldsymbol{N}_{\boldsymbol{i}}$.

4.2.3 TRANSPOSITION OPERATION: For each byte at the index $S_{j}$ in the source image $S$, where $\boldsymbol{j}: 0 \ldots S_{\text {Length }}$. Successively exchange the location of $\boldsymbol{S}_{\boldsymbol{j}}$ with the bytes in $\boldsymbol{S}$ at the indices recoded in the list $\boldsymbol{L}_{j}$. After completing this operation, the original byte at the index $S_{j}$ is transposed to a new location in the source image $\boldsymbol{S}$.

\subsection{LOCAL BLOCK PROCESSING}

To achieve a high level of confusion and diffusion in the image pixels, the source image $\boldsymbol{S}$ and the key $\boldsymbol{K}$ are split into a set of blocks and a set of operations are implemented on each block separately.

4.3.1. PREPARATION OPERATION: Split the source image $S$ into a set of sub-lists of length 256 bytes. Similarly, split the secret key $\boldsymbol{K}$ into the same number of sub-lists of length 256 bytes, too. Where $\boldsymbol{S}_{\boldsymbol{i}}$ and $\boldsymbol{K}_{\boldsymbol{i}}$ are the $\boldsymbol{i}^{\text {th }}$ sub-list of the source image $\boldsymbol{S}$ and the secret key $\boldsymbol{K}$, respectively. It is necessary to be mentioned here that if the number of bytes in the secret key file $\boldsymbol{K}$ is less than the number of bytes in the source image $\boldsymbol{S}$, then the bytes in the secret key file are repeated to match the number of bytes in $\boldsymbol{S}$. Figure 2 shows an example of $\boldsymbol{S}_{\boldsymbol{i}}$ and $\boldsymbol{K}_{\boldsymbol{i}}$ sub-lists.

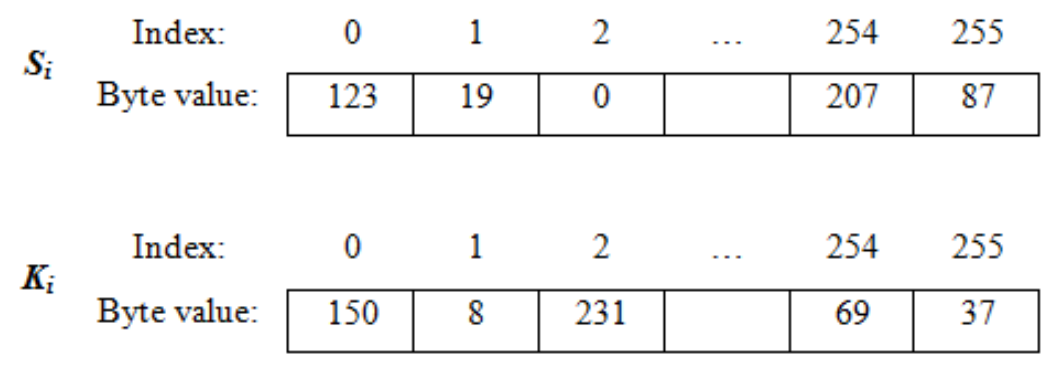

Fig. 2 Examples of $S_{i}$ and $K_{i}$ sub-lists. 


\subsubsection{LOCAL KEY GENERATION (BINARY SEARCH TREE)}

After completing the previous preparation operation, the following steps are implemented (on each block of $\boldsymbol{K}$ ) to generate the Binary search tree (in (1)) that will be used (in (2)) to extract the necessary lists to be used later in the next operations.

1. Read sequentially the distinct byte values (i.e., without repetition) in $\boldsymbol{K}_{i}$. And generate the corresponding Binary search tree from them. The generated Binary search tree must contain 256 nodes; each node contains one value in the range $\mathbf{0}$ to $\mathbf{2 5 5}$. If some bytes haven't been found in $\boldsymbol{K}_{i}$, then these missed values will be inserted into the Binary search tree after finishing the read operation of the byte values in $\boldsymbol{K}_{\boldsymbol{i}}$.

2. Extract the list $\boldsymbol{L}_{\mathbf{0}}$ to $\boldsymbol{L}_{\mathbf{2 5 5}}$ of values in the path of each node from $\boldsymbol{N}_{\mathbf{0}}$ to $\boldsymbol{N}_{\mathbf{2 5 5}}$ in the generated Binary search tree in (1) above.

4.3.3. SUBSTITUTION OPERATION: For each byte $B_{j}$ or the index $j$ of the sub-list $S_{i}$, where $j: 0 \ldots 255$, implement an XOR operation using equation 5 for $B_{j}$ with all the bytes values in the $L_{j}$.

$$
\boldsymbol{B}_{\boldsymbol{j}}=\boldsymbol{B}_{\boldsymbol{j}} \oplus L_{j k}
$$

Where $\oplus$ represents the XOR operation and k represents the values in the list $\boldsymbol{L}_{\boldsymbol{j}}$.

The resulting value of $\boldsymbol{B}_{j}$ replaces the corresponding byte $\boldsymbol{B}_{j}$ in the original image. At the completion of this substitution phase, the encrypted image proceeds next to the transposition phase.

4.3.4. TRANSPOSITION OPERATION: For each byte $B_{j}$ at the index $j$ in the sub-list $S_{i}$, where $j: 0 \ldots 255$, successively exchange the location of $B_{j}$ with the bytes in $S_{i}$ at the indices stored in the list $L_{j}$. Hence, the original byte at the index $B_{j}$ is shifted to a new location in the sub-list $S_{i}$.

\subsection{CONSTRUCT ENCRYPTED IMAGE $E$}

The resulted sub-lists obtained after the transposition phase are merged together in order to construct the encrypted output image $\boldsymbol{E}$, which will be either stored for later use or transmitted over any insecure channel to the intended recipient.

As the decryption process is the inverse of the encryption process, hence, having the encrypted image $\boldsymbol{E}$ as input, and the secret key $\boldsymbol{K}$, the source image $\boldsymbol{S}$ can be produced as the output. Both $\boldsymbol{E}$ and $\boldsymbol{K}$ are treated as files of bytes. The same main operations described in the encryption phase are implemented during the decryption phase with the exception that the substitution and transposition operations are performed in reverse order. Therefore, no need for repeating these processes here.

\section{RESULTS \& DISCUSSION}

To evaluate the proposed encryption method, hundreds of images have been encrypted using different keys. Images of various sizes, contents, and types have been used for testing purposes in order to check the performance of the proposed encryption and decryption processes, looking for advantages, drawbacks, and discrepancies. However, different binary search trees were also experimented with. However, three different source images were selected to be listed in this paper as examples, namely a snappy girl photo (size: $166 \times 256$ ), a bus (size: $533 \times 300$ ), and a camera (size: $455 \times 256$ ). Figure 3 illustrates these selected images. 


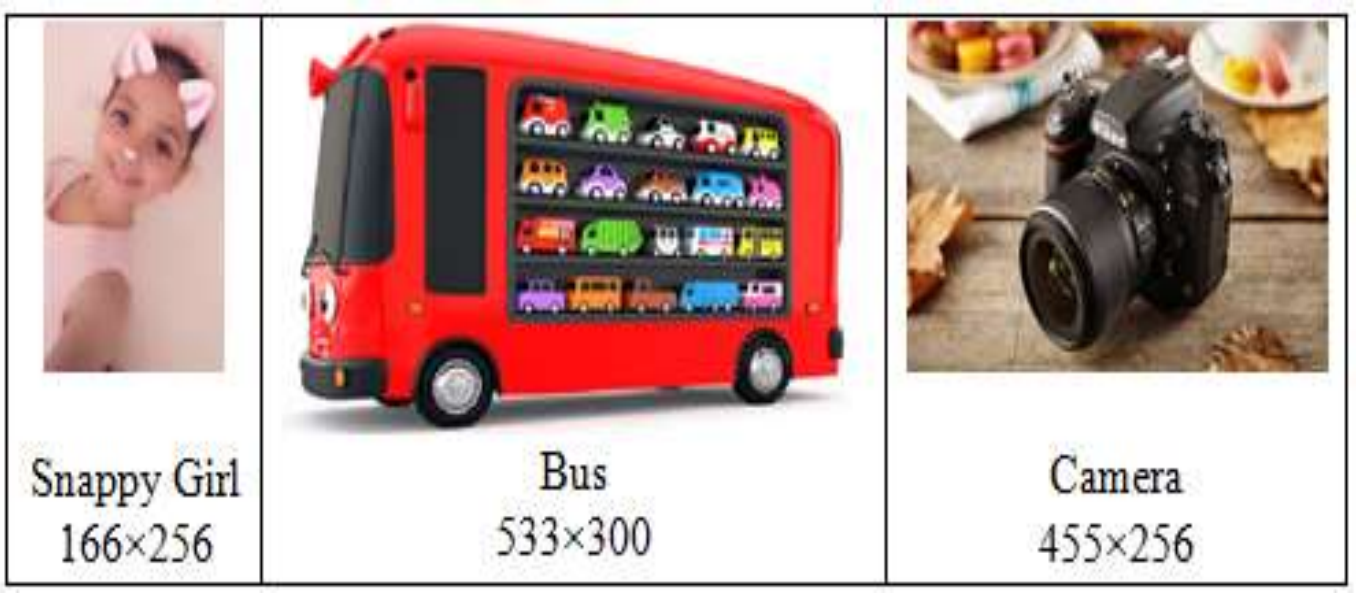

Fig. 3 Selected source images used in the experiments.

The performance observation of the proposed encryption method included Visual and Statistical Tests, Avalanche Effect, Comparison with other Methods, and the key size used. These observations are presented and discussed in the following.

\subsection{VISUAL AND STATISTICAL TESTS}

One of the main factors of the performance for any image encryption method is the distortion ratio of the encrypted images that is generated by the ciphering process. This distortion is clearly noticed for all considered encrypted images. As an example of this factor, Figure 4 shows illustrations for the obtained distortion in the selected images of Figure 3. It is visually clear that the proposed method was successful in producing highly distorted (garbage) encrypted image from the input source image.

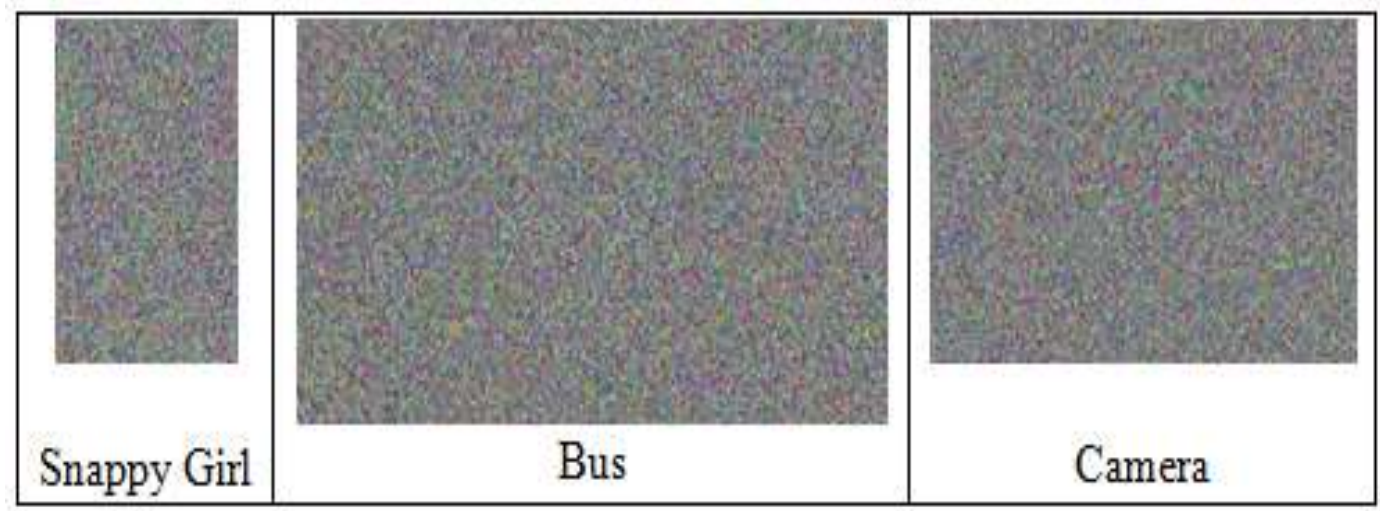

Fig. 4 Corresponding encrypted images for the source images in Fig. 3

Another important factor in the efficiency of any image encryption method is its ability to produce flatness in the histogram of the colors (byte intensity distribution) in the encrypted image compared with a histogram of the colors (byte intensity distribution) in the source image. Figure 5 depicts the bytes intensity distribution histogram for the source and encrypted images of the examples under consideration. 


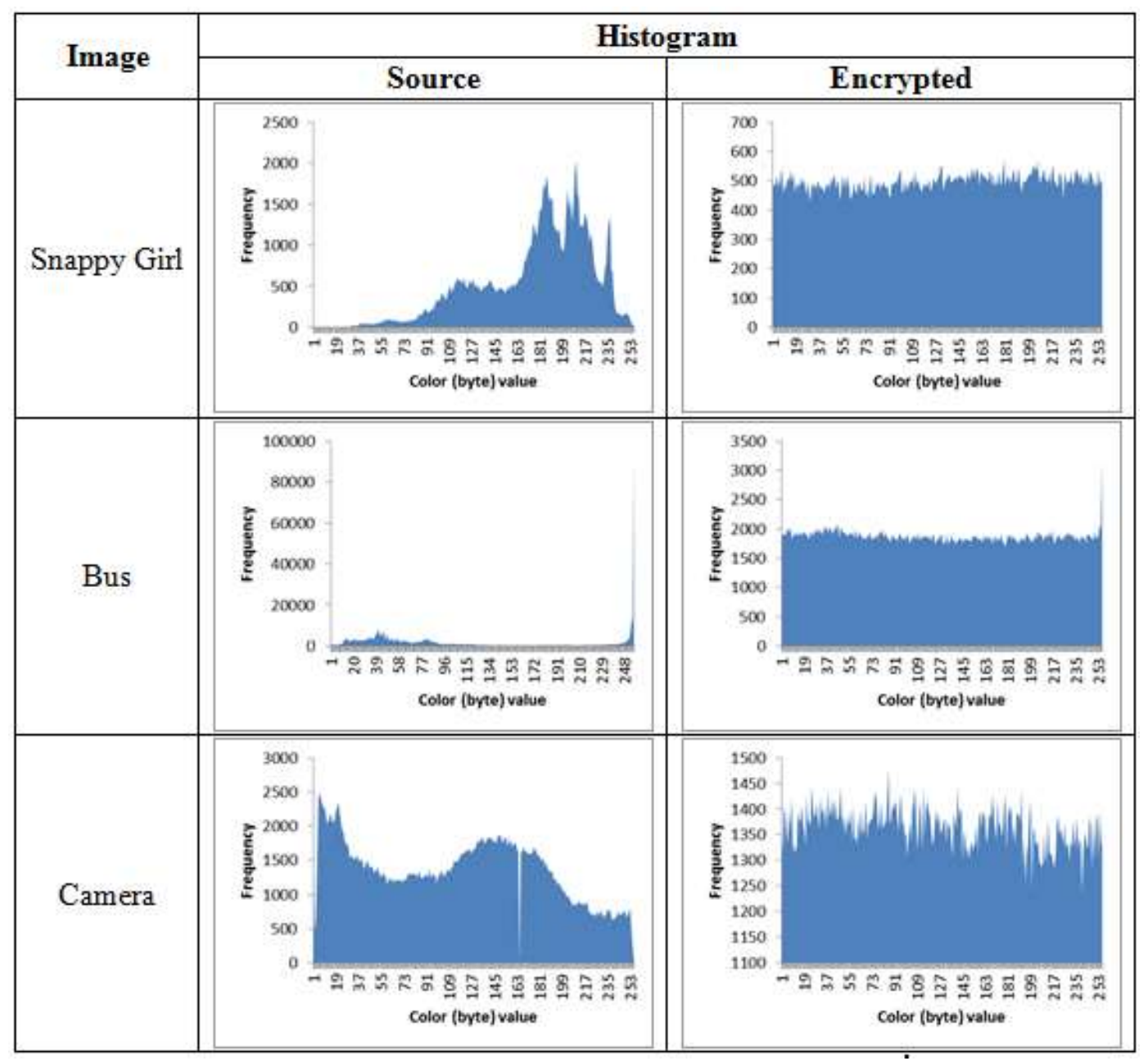

Fig. 5 Histogram of the colors (bytes) of source and encrypted images

Comparing the histograms for the source and encrypted images shows clearly that the histogram of the encrypted images has been widened in such a way that indicate the even distribution of the produced distortion by the proposed method over the whole color range.

\subsection{AVALANCHE EFFECT}

The construction of an encryption technique should take into consideration the avalanche effect as one of the main design objectives. Therefore, it represents one of the desirable properties in any cryptographic algorithm. Avalanche effect reflects the sensitivity of the encryption method even to tiny variations in the parameters; hence it means that when a small change in an input (either in the key or the plaintext) this should cause a tremendous/big change in the cipher text. Any encryption method's success in achieving this property makes it a high-quality encryption method. The following two subsections present the effect of changing bits in the key on the number of bits that will be changed in the generated encrypted image and the recovered source image respectively.

5.2.1. NUMBER OF BITS CHANGED IN THE ENCRYPTED IMAGE: To test the quality of the proposed encryption method and the strength of the used encryption key of avalanche effect, a few bits in the used key are changed during the experiments, then the number of change bits resulted in the encrypted image were calculated according to equation 6. Table II lists the avalanche effect test results that have been recorded during the experiments for different number of changed bits in the encryption key. Although, this 
test is performed for many images, only the results for the Snappy girl image is listed here as an example.

$$
\text { Avalanche Effect }=\frac{\text { Number of changed bits in key used }}{\text { Total number of bits in encrypted image }} \times 100
$$

Table II. Avalanche Test Recorded Results of the Snappy Girl Image.

\begin{tabular}{|c|c|}
\hline $\begin{array}{c}\text { Number of bits changed } \\
\text { in the encryption key }\end{array}$ & $\begin{array}{c}\text { Avalanche Test Value } \\
(\boldsymbol{\%})\end{array}$ \\
\hline 1 & 49.733 \\
\hline 6 & 50.666 \\
\hline 20 & 49.957 \\
\hline 30 & 49.671 \\
\hline
\end{tabular}

To highlight the efficiency of the proposed encryption method compared with the other well-known encryption methods, Table III shows the average values of the avalanche effect test that are computed by equation 6 for the experiments on various color images using the proposed method compared with those calculations for DES and AES encryption methods

Table III. Average of Avalanche Test Values for the Proposed, DES and AES Encryption Methods

\begin{tabular}{|c|c|}
\hline $\begin{array}{c}\text { Encryption } \\
\text { Method }\end{array}$ & $\begin{array}{c}\text { Average of the Avalanche Test } \\
\text { Values (\%) }\end{array}$ \\
\hline Proposed & 51.122 \\
\hline DES & 50.057 \\
\hline AES & 49.970 \\
\hline
\end{tabular}

It can be seen from Tables II and III that the average avalanche effect of the proposed method is acceptable and slightly better as compared with those for DES and AES algorithms.

5.2.2. RECOVERED SOURCE IMAGE: Another way of looking at the avalanche effect by testing the sensitivity of any changes in the key used in the proposed method against any changes in its bits of the decryption key. The avalanche test has been applied to the key, by changing some bits in the decryption key and use it to decrypt the encrypted image. Figure 6 shows the decrypted image after changing a number of bits are in the key. It is clear that when some bits change in the key, the original source image cannot be recovered from the encrypted image. This means that the key used in the proposed method has inherent resistance against the avalanche effect.

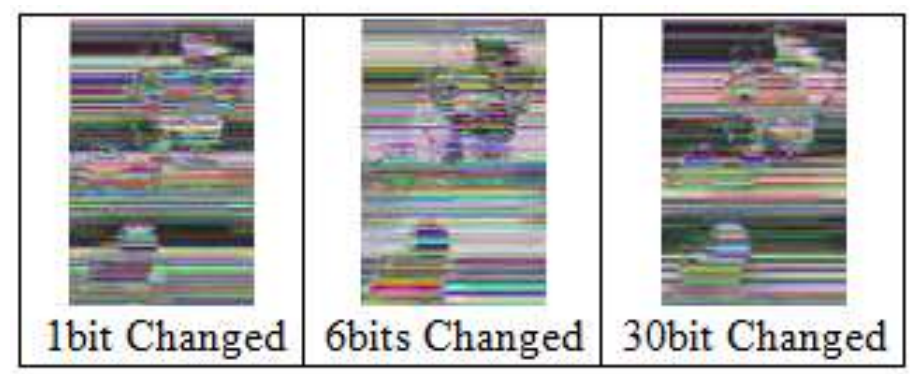

Fig. 6 The recovered source images (of the Snappy girl image) after changing bits in the key 


\subsection{NMAE AND PSNR COMPARISON}

In order to have a more rigorous vision about the performance of any encryption method, a set of metrics such as Normalized Mean Absolute Error (NMAE), Peak Signal to Noise Ratio (PSNR), and Encryption Time (ET) should be computed and compared with the established and widely used methods. Hence, NMAE and PSNR for the proposed Encryption method have been computed using equation 7 and 8 respectively. The obtained results are compared with those for DES and AES algorithms, and are listed in Table IV and Table V. Moreover, the execution time for the encryption process of the proposed method is recorded and compared with its values when executing DES and AES, and the results are listed in Table VI. In order to have a more realistic comparison, all calculation for the three methods were done on the same computing environment.

$$
\begin{gathered}
N M A E=\frac{\sum_{k=0}^{\text {SLength }}|S(k)-E(k)|}{\text { SLength }} \times 100 \\
P S N R_{d b}=10 . \log _{10}\left(\frac{M a x_{S}^{2}}{N M A E}\right)
\end{gathered}
$$

Where MaxS is the maximum possible pixel value of the image $S$.

Table IV. Normalized Mean Absolute Error Values (in \%)

\begin{tabular}{|c|c|c|c|}
\hline \multirow{2}{*}{ Image } & \multicolumn{3}{|c|}{ Method } \\
\cline { 2 - 4 } & Proposed & DES & AES \\
\hline Snappy Girl & 45.40 & 46.03 & 45.89 \\
\hline Bus & 90.07 & 90.38 & 89.98 \\
\hline Camera & 93.00 & 93.05 & 93.25 \\
\hline
\end{tabular}

Table V. Peak Signal to Noise Ratio Values (in decibel dB)

\begin{tabular}{|c|c|c|c|}
\hline \multirow{2}{*}{ Image } & \multicolumn{3}{|c|}{ Method } \\
\cline { 2 - 4 } & Proposed & DES & AES \\
\hline Snappy Girl & 7.67 & 7.60 & 7.62 \\
\hline Bus & 6.27 & 6.29 & 6.31 \\
\hline Camera & 6.86 & 6.86 & 6.85 \\
\hline
\end{tabular}

Table VI. Encryption Time values (in msec.)

\begin{tabular}{|c|c|c|c|}
\hline \multirow{2}{*}{ Image } & \multicolumn{3}{|c|}{ Method } \\
\cline { 2 - 4 } & Proposed & DES & AES \\
\hline Snappy Girl & 360 & 516 & 454 \\
\hline Bus & 288 & 1276 & 1636 \\
\hline Camera & 929 & 854 & 1222 \\
\hline
\end{tabular}

From Table IV and Table V, it can be seen, that the NMAE and PSNR measurements for the proposed encryption method is comparable with those for DES and AES algorithms. This result can be considered as an encouraging sign for the success of the proposed method. Furthermore, the execution time for the proposed method shows remarkable improvement for most images, however, for some images it was much faster than AES but slightly less for DES. These observations can be explained as they are attributed to the image contents or quality of the source image. However, on the average, there is a noticeable improvement in the execution speed of the proposed method.

\subsection{KEY SIZE USED}

The size of the encryption key used in any cryptographic methods plays the major factor in algorithm immunity and the level of difficulty faced by attackers. For the proposed image encryption method, two keys are used, one is used in the global operation and another one 
is used in the local operations. The number of bits in the global key $K_{G}$ and those in the local operation $K_{L}$ are calculated using equation 9 and 10 .

$$
\begin{aligned}
& K_{G}=K_{\text {Length }} \times 8 \\
& K_{L}=256 \times 8
\end{aligned}
$$

The key size that has to be breached by the attacker is the total number of bits in both keys (i.e., Their summation $K_{G}+K_{L}$ ). This key is proportional to the size of the input key selected by the user, and since the user can select a file of any size as the key, obviously, a large enough key will be selected to make life extremely difficult for attackers. Table VII gives a comparison for key size expected for the proposed method with those used by other encryption methods [14].

Table VII. Encryption Key Size Comparison [14]

\begin{tabular}{|l|l|}
\hline Algorithm & Used Key Size (in bit) \\
\hline 3DES & $56,112,168$ \\
\hline AES & 128,192, or 256 \\
\hline Twofish & 128,192, or 256 \\
\hline Blowfish & 32 to 448 \\
\hline RC4 & 40 to 2048 \\
\hline Proposed Method & $>2048$ \\
\hline
\end{tabular}

\section{CONCLUSIONS}

The novel algorithm presented in this paper utilizes the binary search tree as a mean for producing a strong secret encryption key. This key is practically a very long, having two parts; local and global free user-controlled length, hence it has resulted in a key space much larger than globally used algorithms, such as AES and 3DES.

Visually observed encrypted images and histogram energy distribution were satisfactory due to the even distribution of the energy over the color intensity range from 0 to 256.

Moreover, comparison of the measured normalized mean square error, and peak signal to noise ratios for the proposed algorithm were to in good agreements with those for AES and DES, and hence, great promises on the expected usability of this cryptography technique can be anticipated. Besides, a remarkable improvement in the algorithm runs time execution speed.

\section{ACKNOWLEDGEMENTS}

Mohammed A. F. Al-Husainy and Hamza A. A. Al-Sewadi are grateful to the Middle East University Amman (Jordan) for the financial support granted to cover the publication fee of this research article.

\section{REFERENCES}

[1] Schnier B.Applied Cryptography: protocols, algorithms and source code in C. John Wiley \& Sons. 1996. https://mrajacse.files.wordpress.com/2012/01/applied-cryptography-2nd-ed-b-schneier.pdf

[2] William, S. \& Lawrie, B. Computer Security: Principles and Practice. 3rd Ed., Pearson Press. 1976. https://amplio.belluzzifioravanti.it/pluginfile.php/144662/mod_resource/content/1/Computer $\% 20$ Security $\% 20$ Principles $\% 20$ and $\% 20$ Practice $\% 20 \% 282$ nd $\% 20$ Edition $\% 29$.pdf

[3] Whitfield, D. W. and Hellman, M. E. "New Directions in Cryptography." IEEE Transactions on Information Theory, It-22.6, (1976). 644-654. http://www.cs.utexas.edu/users/shmat/courses/cs380s/dh.pdf

[4] Tripathi, R. \& Agrawal, S. "Comparative Study of Symmetric and Asymmetric Cryptography Techniques." International Journal of Advance Foundation and Research in Computer (IJAFRC), 1.6. (2014). 68-76. 
https://pdfs.semanticscholar.org/e0e4/810c5276f9c05cc82425fcf911f206c52bef.pdf

[5] Lim, H. and Mun, J. H. "High-speed packet classification using binary search on length." Proceedings of the 3rd ACM/IEEE Symposium on Architecture for networking and communications systems (ANCS'07). (2007). 137-144.

https://www.researchgate.net/publication/221046084_High-

Speed_Packet_Classification_Using_Binary_Search_on_Length

[6] Wei R., and Zebg Z. "KIST: A new encryption algorithm based on splay." IACR Cryptology ePrint Archive. (2010).

https://www.researchgate.net/publication/220335336_KIST_A_new_encryption_algorithm_b ased_on_splay

[7] Saraswathi, P. V. and Venkatesulu, M. "Block Cipher Algorithm for Multimedia Content Protection with Random Substitution using Binary Tree Traversal.” Science Publications, Journal of Computer Science, 8.9. (2012). 1541-1546.

http://citeseerx.ist.psu.edu/viewdoc/summary?doi=10.1.1.686.652

[8] Vanaga, A. "Efficient Cryptography Technique for Data Security using Binary Tree." International Journal of Innovative Research in Computer and communication Engineering, 2(Special Issue), (2014). 67-76. http://www.ijircce.com/upload/2014/sacaim/10_Paper\%205.pdf

[9] Sharma, S. and Bhatt, S. "Encryption of Message Block using Binary Tree in Block Cipher System: An Approach.” IJSTE - International Journal of Science Technology \& Engineering, 2.1. (2015). https://www.academia.edu/15895315/Encryption_of_Message_Block_using_Binary_Tree_in_ Block_Cipher_System_An_Approach

[10] Sagar, L. E. C. V. "Data Security Using Tree Traversal. Global Journal of Computer Science and Technology.” Software \& Data Engineering, 5.3, Version 1.0, Global Journals Inc. (USA). (2015). file:///C:/Users/GTS/Downloads/1170-1-1178-1-10-20150714.pdf

[11] Priya, A., Sinha K., Darshan, M. P., and Sahana, S. K. "A Novel Multimedia Encryption and Decryption Technique Using Binary Tree Traversal." Proceeding of the Second International Conference on Microelectronics, Computing and Communication Systems (MCCS 2017). (2017). 163-178.

[12] Sivakumar T. Humshavarthini, K., Jayasree, M., and Eswaran, M. "Data Encryption Using Binary Tree Traversal (DEBTT).” International Journal of Advanced Technology in Engineering and Science, 5.4. (2017). 353-362. https://www.ijates.com/images/short_pdf/1491493867_IF2028ijates.pdf

[13] Amounas, F. “A New Encryption Algorithm to Increase Security of Amazigh Text through Tree Traversal Technique." International Journal on Recent and Innovation Trends in Computing and Communication (IJRITCC), 5.1, . (2017). $217-222$.

http://www.ijritcc.org/download/browse/Volume_5_Issues/January_17_Volume_5_Issue_1/1 485582293_27-01-2017.pdf

[14] Ritu, T., and Sanjay, A. "Comparative Study of Symmetric and Asymmetric Cryptography Techniques." International Journal of Advance Foundation and Research in Computer (IJAFRC), 1.6. (2014). 68-76. https://pdfs.semanticscholar.org/e0e4/810c5276f9c05cc82425fcf911f206c52bef.pdf 